\title{
Effects of Silk Fibroin Moisturizing Cream on Acetone/Ether-Induced Dry Skin Pruritus in Mice
}

\author{
Jianming $\mathrm{He}^{{ }^{*}}$, Yuling Tong² \\ ${ }^{1}$ Project Department, Zhejiang Wolwo Bio-Pharmaceutical Co., LTD., Shanghai, China \\ ${ }^{2}$ Marketing Department, Zhejiang Wolwo Bio-Pharmaceutical Co., LTD., Shanghai, China \\ Email: ^hejianming@wolwobiotech.com
}

How to cite this paper: He, J.M. and Tong, Y.L. (2019) Effects of Silk Fibroin Moisturizing Cream on Acetone/Ether-Induced Dry Skin Pruritus in Mice. Journal of Cosmetics, Dermatological Sciences and Applications, 9, 235-243.

https://doi.org/10.4236/jcdsa.2019.93021

Received: July 31, 2019

Accepted: September 16, 2019

Published: September 19, 2019

Copyright $\odot 2019$ by author(s) and Scientific Research Publishing Inc. This work is licensed under the Creative Commons Attribution International License (CC BY 4.0).

http://creativecommons.org/licenses/by/4.0/

(c) () Open Access

\begin{abstract}
Objectives: The morbidity of dry and itchy skin was high during a person's lifetime and it had a significant negative impact. This study aimed to investigate the positive effect of silk fibroin moisturizing cream on dry skin pruritus induced by acetone/ether-water (AEW) method in mice. Method: We established a dry skin model in mice with AEW treatment, and the control group was treated with ultrapure water. The mice in AEW model group were locally administrated with silk fibroin moisturizing cream (SilkArmor)'. The transepidermal water loss (TEWL), scratching behavior, condition of skin erythema, skin histology (by hematoxylin-eosin staining) and the results of electron microscopy were measured to evaluate the positive effect of SilkArmor on dry and itchy skin in mice. Results: The TEWL value, spontaneous scratching behavior and erythema score were significantly increased after AEW modeling compared with the control group. There was no significant difference in the TEWL value, scratching behavior and erythema score between the SilkArmor group and the control group $(P>0.05)$. Compared with the AEW group, the value of TEWL, scratching behavior and the erythema score of mice in the AEW + SilkArmor group were improved, with statistically significant differences $(P<0.05)$. Skin histopathology and electron microscopy also showed that SilkArmor had certain improvement and repairment on AEW-induced skin pathological changes. Conclusions: A dry skin model was successfully established in this experiment, showing that SilkArmor brought an obvious alleviation to AEW-induced dry and itchy skin in mice.
\end{abstract}

\section{Keywords}

Dry, Pruritus, Silk Fibroin, SilkArmor, Moisturized 


\section{Introduction}

Pruritus is a common unpleasant sensation and a characteristic feature of various skin diseases, which has significant negative impacts on the quality of patients' life [1] [2]. In addition, some non-dermatological diseases such as chronic renal failure [3], cholestasis [4], and diabetes [5] can also induce itchy skin. However, the mechanism of itch has not been fully discovered, but lots of elements including genetic, environmental and immune factors are the dangerous elements of pruritus [6]. In particular, dry skin, which is associated with incomplete cuticle and disrupted epidermal barrier, could be one of the triggering factors of pruritus [7]. Thus, the method of alleviating skin dryness and rebuilding the skin barrier is thought to be an important therapeutic treatment for pruritus.

Moisturizers can reduce skin moisture loss and repair epidermal dysfunction. Applying moisturizing cosmetics can not only protect healthy and normal skin, but also repair damaged skin [8]. Silk fibroin, obtained from silk, has a close affinity to human skin and can moisturize skin, because it is rich in various amino acids and trace elements [9]. Also, silk fibroin can increase the moisture content of the cuticle, promote the synthesis of collagen and repair the barrier dysfunction of the skin epidermis, which results in relieving dry and itchy skin [10]. Studies also have shown that silk fibroin can not only replenish water of skin but also prevent it from evaporation [11]. Silk fibroin moisturizing cream (SilkArmor) ${ }^{\circ}$ is rich in water-soluble and high molecular weight silk fibroin, with good moisturizing function. In this study, we successfully established an acetone/ether-water (AEW) model on ICR mice to induce dry and itchy skin. And we aimed at evaluating the positive effect of SilkArmor on AEW-induced dry and itchy skin by measuring the transepidermal water loss (TEWL), scratching behavior, skin erythema, skin histology and the results of electron microscopy.

\section{Materials and Methods}

\subsection{Materials}

Twenty-eight six-week-old male ICR mice were purchased from Shanghai Jiesijie Experimental Animals Co., LTD. (Shanghai, China). Acetone and ether are analytical pure reagents, purchased from Sinopharm Chemical Reagents Co., Ltd. (Shanghai, China). The ultrapure water was made of ultrapure water systems (Cascada I, PALL, New York, USA); Meanwhile, silk fibroin moisturizing cream (SilkArmor) ${ }^{\circledR}$ was researched and developed by Zhejiang Wolwo Bio-pharmaceutical Co., Ltd.

\subsection{Methods}

\subsubsection{Animals}

All ICR mice were maintained with a $12 \mathrm{~h}$ light/dark cycle and kept at standard room temperature $\left(22^{\circ} \mathrm{C} \pm 2{ }^{\circ} \mathrm{C}\right)$ and humidity $(55 \% \pm 10 \%)$, with enough food and water available. All mice were fed to adapt to the environment for three days and then conducted to experiment. Before the experiment, mice were divided 
randomly into four groups: the control group $(\mathrm{n}=6)$, the SilkArmor group $(\mathrm{n}=$ $6)$, the AEW group $(n=6)$, and the AEW + SilkArmor group $(n=10)$. The hair on the neck and back of the mice was carefully removed with a hair shaver after anesthesia, and the skin was completely exposed at least 1 day before modeling. All animal study protocols were designed according to guidelines set by the $\mathrm{Na}$ tional Institute of Health Guide for the Care and Use of Laboratory Animals, and approved by the Animal Ethical Experimentation Committee.

\subsubsection{Establishment of AEW Model}

To establish itch of dry skin on the neck and back of mice, we followed a previously-reported method [12] [13]. Briefly, the hair of the neck and back of mice was shaved to expose the skin 1 day before the treatments. The itch of dry skin was induced by the treatment with acetone/ether (1:1 mixture) for $20 \mathrm{~s}$, followed by ultra-pure water for $30 \mathrm{~s}$. For the control group, the exposed skin of mice was treated with ultra-pure water only for $50 \mathrm{~s}$. The mice in the SilkArmor group were coated with SilkArmor after 30 min of ultra-pure water treatment, and the mice in the AEW + SilkArmor group were administrated with SilkArmor after AEW modeling for $30 \mathrm{~min}$. The above operation was repeated at 9 am and $4 \mathrm{pm}$, twice a day for 6 consecutive days. Crucially, the value of TEWL, scratching behavior and the erythema score of mice in each group were recorded detailly and videotaped every day. At last, the mice were sacrificed to collect the skin samples on the seventh day.

\subsubsection{Sample Preparation for Histological Observation}

Mice in each group were sacrificed by breaking the neck and then fixed. The skin tissue was removed via ophthalmic scissors, fixed with $4 \%$ paraformaldehyde solution, dehydrated routinely, transparentized, embedded, sliced, stained with hematoxylin-eosin (HE), and sealed.

\subsubsection{Sample Preparation for SEM}

The skin tissue was removed via ophthalmic scissors, followed by fixing on filter paper. The sample was cleaned by phosphate buffer solution, fixed with $2.5 \%$ glutaraldehyde solution, dehydration with ethanol, replaced, freeze-dried and sprayed gold before observation.

\subsection{Pharmacodynamics Indexes}

\subsubsection{Measurement of TEWL}

TEWL value is one of the most common indicators to detect the function of skin barrier, the higher TEWL value indicates the worse function of skin barrier and the more severe damage of skin [14]. To evaluate skin barrier function, TEWL of each mouse was measured via the transdermal water loss meter (UAPOMETER, Delfin Technologies Led., Kuopio, Finland) and each parameter was measured at 8:10 am on the second day after the final dermal barrier destruction treatment.

\subsubsection{Analysis of Scratching Behavior}

Before measurement of scratching behavior, mice were placed in a transparent 
cage with the opaque middle clapboard to prevent the interference between each other. The behavior of mice was recorded by WiFi camera (cs-c2c-1A1WFR, Hangzhou, China). The mice were kept doors and windows closed to reduce interference. The number of spontaneous scratching behavior was counted to evaluate the degree of pruritus after the mice were put into the cage for $10 \mathrm{~min}$ and until the cumulative activity time reached $30 \mathrm{~min}$. One scratch refers to the claw of mouse scratching the exposed skin for one or many times, ending with the claw landing or licking. A scratch with the forepaw was counted as an invalid scratch.

\subsubsection{Evaluation of Skin Erythema}

The damaged skin of mice in each group was recorded, scored and photographed in detail. The scoring criteria were shown as bellow (Table 1) [15].

\subsubsection{Histological Observation}

The stained tissue samples were observed the epidermal thickness of epithelial tissue, the hyperplasia of the stratum corneum and the inflammatory infiltration of epithelial tissues, via the digital pathological section scanning system (Pannoramic MIDI, 3D HISTECH, Hungary).

\subsubsection{Observation of Electron Microscopy}

After sample preparation, the skin tissue was observed via the high resolution field emission scanning electron microscope (Sirion 200, Thermo Fisher Scientific, USA).

\subsection{Statistical Analysis}

Statistical analysis of the data sets was carried out via SPSS 22.0. The data were repeated 3 times for each group and each value was presented as means \pm SD. The statistical significance was analyzed by two-way repeated measures analysis of variance (ANOVA). $P$ value of less than 0.05 was considered statistically significant.

\section{Results}

\subsection{The Value of TEWL}

In this study, AEW was used to establish the model of dry skin pruritus in mice. As shown in Figure 1(a), there was no obvious difference on the TEWL value

Table 1. The erythema scoring criteria of skin irritation response.

\begin{tabular}{cc}
\hline Skin irritation reaction & Score \\
\hline No erythema & 0 \\
Mild erythema (barely visible) & 1 \\
Obvious erythema & 2 \\
Moderate to severe erythema & 3 \\
Severe erythema (mild scab) to mild eschar (deep lesion) & 4 \\
\hline
\end{tabular}



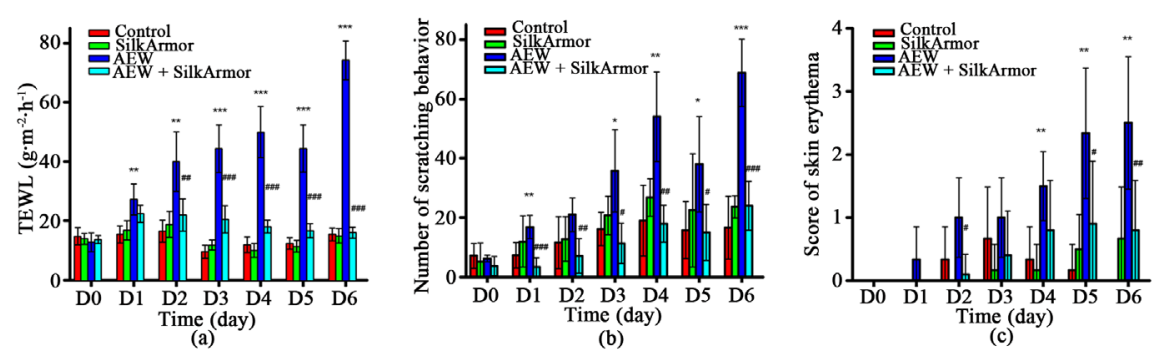

Figure 1. Effects of SilkArmor on (a) TEWL; (b) scratching behavior; and (C) skin erythema induced by AEW in mice. Each value represents the mean \pm SD. Compared with the control group, ${ }^{\star} P<0.5,{ }^{\star *} P<0.1,{ }^{* *} P<0.001$. Compared with the AEW group, ${ }^{*} P<$ $0.5,{ }^{\# \#} P<0.1,{ }^{\# \# \#} P<0.001$.

between the control group and the SilkArmor group during the whole period of the experiment. On the contrary, the TEWL value of mice in the AEW group was significantly higher than that in the control group from the first day of modeling. Compared with the AEW group, the TEWL value of mice in the AEW + SilkArmor group significantly decreased since the second day treated with SilkArmor.

\subsection{Spontaneous Scratching Behavior}

The degree of pruritus was evaluated by the number of spontaneous scratching behavior of mice. The results of ethology showed that, compared with the control group, there was no significant difference on the scratching number of mice in the SilkArmor group, but the scratching number of mice in the AEW group were obvious bigger on the first day and after the third day after modeling (Figure 1(b)). Meanwhile, the number of scratching actions of mice induced by AEW treatment was significantly reduced in the AEW + SilkArmor group from the first day when compared to the AEW group, which has statistical significance.

\subsection{Condition of Skin Damage}

The skin lesions of mice in each group was evaluated by detecting the score of skin erythema and the higher erythema score indicated more severe injury of skin. Similar to the other results, there was no obvious difference of the erythema score between the mice in the control group and the SilkArmor group (Figure 1(c)). After modeling, the score of skin erythema was increased significantly after the fourth day in the AEW group, indicating the damage of skin. Compared with the AEW group, the score of skin erythema in the AEW + SilkArmor group started to show a significant decrease on the fifth day.

As seen in Figure 2, the neck and back skin of mouse in the AEW group displayed obvious redness, erythema and scab on the sixth day. While in the control group and the SilkArmor group, the skin of mice was normal and without erythema. At the same time, the skin of mice in the AEW + SilkArmor group showed slight erythema, but the damage of skin was slighter than that of AEW group. 
Control
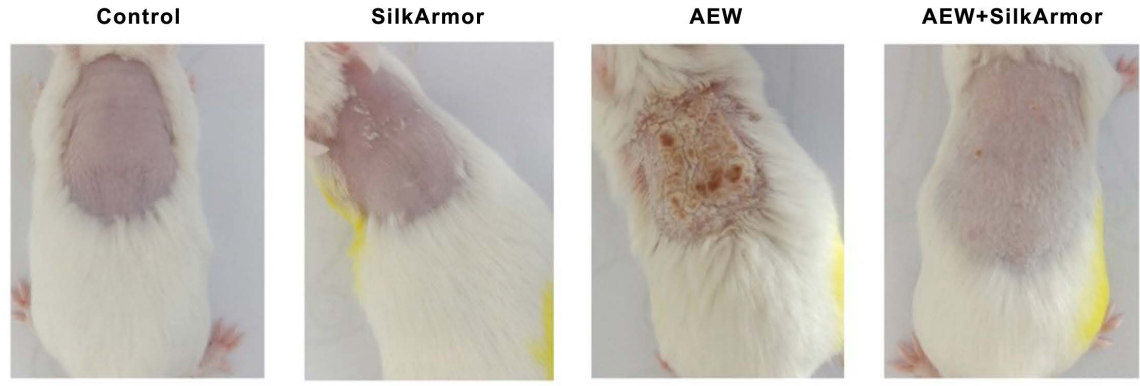

Figure 2. Skin condition of the neck and back of mice in each group on the sixth day.

\subsection{Pathological Changes of Skin Tissue}

In the control group and the SilkArmor group, the structure of the epidermis in the back skin of mice was intact and the skin histopathology showed no obvious change (Figure 3(a) and Figure 3(b)). On the contrary, due to the increase of scratching behavior, the mice in AEW group had obvious epidermal injury or keratinization, as well as inflammatory cell infiltration, resulting in corneous thickening. (Figure 3(c)). Obviously, the structure of skin improved, manifesting in the repair of the cuticle structure and the integrity of the epidermis in the AEW + SilkArmor group (Figure 3(d)).

\subsection{Scanning Results of Electron Microscopy}

The skin epidermis of mice in the control group was intact while that of mice in the AEW group showed fault in the skin epidermis, manifesting as cuticle hyperkeratosis, local thickening and incomplete surface. Meaningfully, the skin of mice in the AEW + SilkArmor group was improved and the epidermis was intact (Figure 4).

\section{Discussion}

The dry and itchy skin has a high morbidity in the population, which can greatly affect physical and mental health of patients and affect their quality of life [16]. The reason for inducing dry skin pruritus may be various internal or external factors, resulting in the epidermal barrier dysfunction. Exactly, restoring the skin barrier function is the best choice to deal with skin problems, and the use of moisturizing cosmetics can repair the barrier function of epidermis, relieve skin dryness and water shortage, and alleviate itch caused by dry skin. In this study, increased TEWL value, spontaneous scratching actions and erythema score in the AEW group were significant, compared with the control group. Meanwhile, the results of skin histopathology and electron microscopy showed the incomplete keratinized and locally thickened cuticular of mice in the AEW group. In conclusion, the mice in the AEW group developed skin barrier dysfunction, increased TEWL, and resulted in increased skin itching, indicating the successful model of dry skin pruritus. Besides, there was no significant difference in each index between the control group and the SilkArmor group, revealing that SilkArmor had no significant adverse effect on normal and healthy skin. 


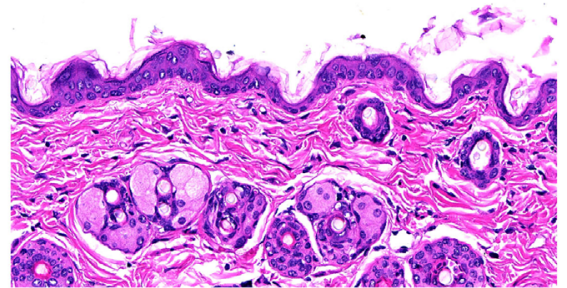

(a)

(

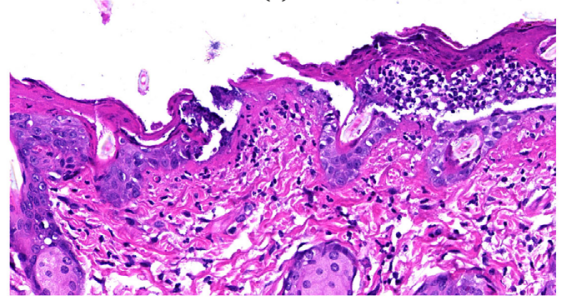

(c)

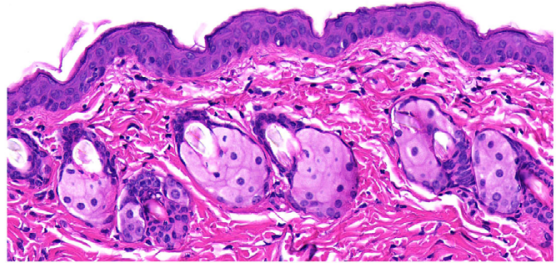

(b)

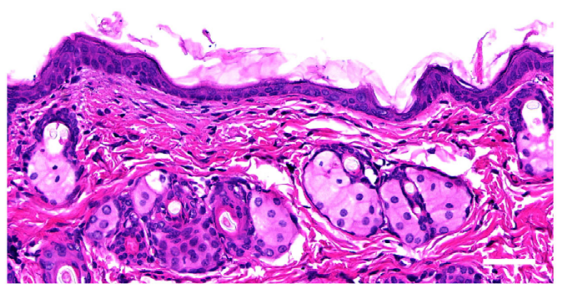

(d)

Figure 3. The structure of the epidermis in the back skin of mice in the (a) control; (b) SilkArmor; (c) AEW and (d) AEW + SilkArmor group after AEW modeling (HE $\times 400)$.

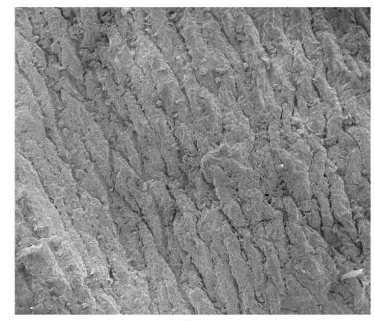

(a)

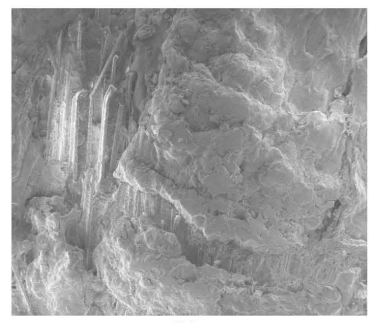

(b)

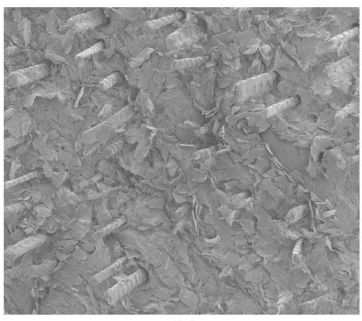

(c)

Figure 4. The scanning electron micrograph of skin on the back of mice in the (a) control; (b) AEW and (c) AEW + SilkArmor group $(\mathrm{HE} \times 200)$.

TEWL value is a common indicator to evaluate the functional barrier of skin. In SilkArmor treated mice, the level of TEWL had a tendency to decrease compared to the AEW group, indicating that the local administration of SilkArmor can significantly reduce the skin moisture loss caused by AEW and improve the situation of hydropenic and dry skin.

After modeling, the level of TEWL increased, and the dehydrated and dry skin led to pruritus. Normally, the number of spontaneous scratching was used to assess the degree of pruritus [17]. On the first day after smeared SilkArmor, the number of spontaneous scratching behavior started to decrease, reflecting a lower itch degree of mice.

The results of skin damage experiment showed that there was obvious erythema and eschar on the skin after 6 days of modeling, and the erythema score was significantly higher than that of the control group, indicating the skin of modeling was significantly damaged. After applying SilkArmor to the skin of AEW modeling, the erythema score significantly decreased after 5 days of application, indicating that SilkArmor can visibly alleviate the damage of AEW to the skin. Also, the photos of skin on the sixth day only showed slight erythema of mice coated with SilkArmor, with lower degree of skin damage than the AEW 
mice.

From the results of the histopathological test and electron microscopy, the application of SilkArmor can reduce the damage to skin, restore corneous layer and rebuild epidermis structure of skin. Possibly, through repairing the stratum corneum, rebuilding epidermis structure of damaged skin and restoring skin barrier dysfunction, SilkArmor reduces the loss of skin moisture and improves the situation of dry and itchy skin.

\section{Conclusion}

In conclusion, SilkArmor can obviously repair the dry skin pruritus induced by AEW in mice. It can repair the damaged skin and deal with the dysfunction of skin barrier, which has an obvious effect on moisturizing and relieving itching. The main active ingredient of SilkArmor is silk fibroin, which may play a key role in the positive effect of dry and pruritus skin, but still needs to be further studied.

\section{Conflicts of Interest}

The authors declare no conflicts of interest.

\section{References}

[1] Okawa, T., Yamaguchi, Y., Takada, S., Sakai, Y., Numata, N., Nakamura F., et al. (2012) Oral Administration of Collagen Tripeptide Improves Dryness and Pruritus in the Acetone-Induced Dry Skin Model. Journal of Dermatological Science, 66, 136-143. https://doi.org/10.1016/j.jdermsci.2012.02.004

[2] Akiyama, T., Carstens, M.I., Piecha, D., Steppan, S. and Carstens, E. (2015) Nalfurafine Suppresses Pruritogen- and Touch-Evoked Scratching Behavior in Models of Acute and Chronic Itch in Mice. Acta Dermato-Venereologica, 95, 147-150. https://doi.org/10.2340/00015555-1879

[3] Combs, S.A., Teixeira, J.P. and Germain, M.J. (2015) Pruritus in Kidney Disease. Seminars in Nephrology, 35, 383-391. https://doi.org/10.1016/j.semnephrol.2015.06.009

[4] Bolier, A.R., Peri, S., Oude Elferink, R.P.J. and Beuers, U. (2012) The Challenge of Cholestatic Pruritus. Acta Gastroenterological Belgica, 75, 399-404.

[5] Yamaoka, H., Sasaki, H., Yamasaki, H., Ogawa, K., Ohta, T., Furuta, H., et al. (2010) Truncal Pruritus of Unknown Origin May Be a Symptom of Diabetic Polyneuropathy. Diabetes Care, 33, 150-155. https://doi.org/10.2337/dc09-0632

[6] Valdes-Rodriguez, R., Stull, C. and Yosipovitch, G. (2015) Chronic Pruritus in the Elderly: Patho-Physiology, Diagnosis and Management. Drug \& Aging, 32, 201-215. https://doi.org/10.1007/s40266-015-0246-0

[7] Fujii, M., Shimizu, T., Nakamura, T., Endo, F., Kohno, S. and Nabe, T. (2011) Inhibitory Effect of Chitosan-Containing Lotion on Scratching Response of Hairless mice with Atopic Dermatitis-Like Dry Skin. Biological and Pharmaceutical Bulletin, 34, 1890-1894.

[8] Jonatan, D.L. and Maria, B. (2015) Clinical Effectiveness of Moisturizers in Atopic Dermatitis and Related Disorders: A Systematic Review. American Journal of Clinical Dermatology, 16, 341-359. https://doi.org/10.1007/s40257-015-0146-4 
[9] Daithankar, A.V., Padamwar, M.N., Pisal, S.S., Paradkar, A.R. and Mahadik, K.R. (2005) Moisturizing Efficiency of Silk Protein Hydrolysate: Silk Fibroin. Indian Journal of Biotechnology, 4, 115-121. https://doi.org/10.1111/j.1473-2165.2005.00200.x

[10] Padamwar, M.N., Pawar, A.P., Daithankar, A.V. and Mahadik, K.R. (2005) Silk Sericin as a Moisturizer: An in Vivo Study. Journal of Cosmetic Dermatology, 4, 250-257. https://doi.org/10.1111/j.1473-2165.2005.00200.x

[11] Zhao, L., Xie, Y., Zheng, Y., Cai, C. and Xiao, H. (2012) Progress in Research Work with Respect to Application of Silk Protein in Cosmetics. China Surfactant Detergent \& Cosmetics, 42, 452-456.

[12] Zhou, F.M., Cheng, R.X., Wang, S., Huang, Y., Gao, Y.J., Zhou, Y., et al. (2017) Antioxidants Attenuate Acute and Chronic Itch: Peripheral and Central Mechanisms of Oxidative Stress in Pruritus. Neuroscience Bulletin, 33, 423-435. https://doi.org/10.1007/s12264-016-0076-Z

[13] Yu, G., Yang, N., Li, F., Chen, M., Guo, C.J., Wang, C., et al. (2016) Enhanced Itch Elicited by Capsaicin in a Chronic Itch Model. Molecular Pain, 12. https://doi.org/10.1177/1744806916645349

[14] Eissa, A. and Diamandis, E.P. (2008) Human Tissue Kallikreins as Promiscuous Modulators of Homeostatic Skin Barrier Functions. Biological Chemistry, 389, 669-680. https://doi.org/10.1515/BC.2008.079

[15] National Health Commission of the People's Republic of China (2005) Technical Specification for Chemical Toxicity Identification.

http://www.nhc.gov.cn/zhjcj/s7885/200804/db2728e69a9845668bd29c4a54bac6f6.sh $\underline{\mathrm{tml}}$

[16] Matterne, U., Apfelbacher, C.J., Vogelgsang, L., Loerbroks, A. and Weisshaar, E. (2013) Incidence and Determinants of Chronic Pruritus: A Population-Based Cohort Study. Acta Dermmato-Venereological, 93, 532-537. https://doi.org/10.2340/00015555-1572

[17] Cao, L.F., Si, M., Huang, Y., Chen, L.H., Peng, X.Y., Qin, Y.Q., et al. (2017) Long-Term Anti-Itch Effect of Botulinum Neurotoxin A Is Associated with Downregulation of TRPV1 and TRPA1 in the Dorsal Root Ganglia in Mice. NeuroReport, 28, 518-526. https://doi.org/10.1097/WNR.0000000000000779 